\title{
$\longrightarrow$
}

\author{
ПСИХОЛОГИЯ РАЗВИТИЯ, АКМЕОЛОГИЯ \\ DEVELOPMENTAL PSYCHOLOGY, ACMEOLOGY
}

\section{ПРИМЕНЕНИЕ КОМПЬЮТЕРНЫХ ИГРОВЫХ ТЕХНОЛОГИЙ ДЛЯ РАЗВИТИЯ РЕГУЛЯТОРНЫХ ФУНКЦИЙ ДОШКОЛЬНИКОВ}

\author{
Александр Н. Веракса*, Дарья А. Бухаленкова \\ Московский государственный университет имени М. В. Ломоносова, \\ 2. Москва, Российская Федерачия \\ "E-mail: veraksa@yandex.ru
}

Работа выполнена при поддержке Гранта Президента МД-441.2017.6

Введение. Авторами показывается актуальность использования и разработки компьютерных приложений для детей дошкольного возраста с иелью развития регуляторных функций. В работе впервые представлен обзор зарубежных исследований, посвященных компьютерным игровым методам развития регуляторных функций.

Теоретическое обоснование. В статье описывается модель регуляторных функций А. Мияке, а также культурно-исторический подход, на который опирались авторы при проведении теоретического исследования.

Результаты. Данный раздел включает рассмотрение основных причин популярности использования и разработки развивающих компьютерных игр и их преимуществ перед стандартными методами: наличие данных о развивающем эффекте существующих игр, о положительном влиянии такого формата тренировочных заданий на мотивачию, возможность гибко адаптировать игру под уровень тренирующегося и более высокая экологичность выполняемыхзаданий. В обзоре проанализированы существуюшие программы и приложения по развитию регуляторных функций у детей дошкольного возраста, требующие участия родителей или воспитателей в процессе тренировки, а также направленные на организацию взаимодействия сверстников. Также в статье рассмотрены основные принципы создания развивающих игр, способных не только адаптироваться к уровню развития пользователя, но и поддерживать необходимую мотивацию во время тренировки регуляторных функций. В исследованиях, посвященных созданию развивающих видеоигр, подчеркивается значение заучивания испытуемым определенных 
наборов действий и применение их в соответствующей ситуации для развития памяти и процессов торможения, а также значимость заданий на скорость для развития когнитивной гибкости.

Обсуждение результатов. Авторы сопоставляют проанализированные разработки в области компьютерных игровых методов и выделяют наиболее значимые черты, способные оказывать развивающее воздействие на регуляторные функции в дошкольном возрасте, что может стать основанием как для оценки уже имеющихся приложений, так и для разработки новых компьютерных видеоигр, направленных на развитие регуляторных функций.

Ключевые слова: регуляторные функции, рабочая память, когнитивная гибкость, сдерживающий контроль, саморегулячия, рефлексия, игра, компьютерные приложения, видеоигры, дошкольный возраст

\section{Основные положения:}

> большинство существующих и применяющихся развивающих программ основываются на идее тренировки регуляторных функций, а не формирования внутренних средств управления собственным поведением, что является основной причиной отсутствия переноса полученных навыков на другие виды деятельности;

> наиболее перспективным направлением развития регуляторных функций представляется разработка таких игровых методов, которые способны адаптироваться под уровень развития дошкольника, создавая зону ближайшего развития;

> многие приложения и развивающие программы предполагают участие родителей или учителей в развитии регуляторных функций, однако не менее перспективным представляется подход, предполагающий сотрудничество детей друг с другом, в котором один ребенок выполняет роль учителя для другого.

Для цитирования: Веракса А.Н., Бухаленкова Д. А. Применение компьютерных игровых технологий для развития регуляторных функций дошкольников // Российский психологический журнал. - 2017. - Т. 14, № 3. - С. 106-132. 


\title{
COMPUTER GAME-BASED TECHNOLOGY IN THE DEVELOPMENT OF PRESCHOOLERS' EXECUTIVE FUNCTIONS
}

\author{
Aleksandr N. Veraksa*, Daria A. Bukhalenkova \\ Lomonosov Moscow State University, Moscow, Russian Federation \\ Correspondence author. E-mail: veraksa@yandex.ru
}

\section{Acknowledgments}

Supported by the presidential grant, project no. MD-441.2017.6

Introduction. Computer applications are important for the development of preschoolers' executive functions. This paper is the first study to overview foreign studies of computer game-based methods for the development of executive functions. Theoretical Basis. The study describes (a) A. Miyake's model of executive functions and (b) the cultural-historical approach.

Results. The study revealed the main reasons for the use and development of serious videogames. Their advantages over standard methods were as follows: (a) Some data indicated the developmental effect of the existing games. (b) Such a format of training tasks had a positive impact on motivation. (c) The game adapted flexibly to a trainee's level. (d) Such tasks had a high ecological compatibility. The review analyzed the existing programs and applications for the development of preschoolers' executive functions, which required the participation of parents or teachers in the process of training and organized peer interaction. The paper described the basic principles of creating serious games, which can not only adapt to the user's development level, but also maintain motivation when training executive functions. The literature on the creation of serious video games showed the importance of a subject's learning of certain sets of actions and their use in appropriate situations for the development of memory and inhibition. Timely actions were important for the development of cognitive flexibility.

Discussion. The comparison of the analyzed computer game-based methods made it possible to distinguish the most significant features, which can develop executive functions at preschool age. The findings can be successfully used for evaluating the existing applications and creating new videogames, aimed at the development of executive functions.

Keywords: executive functions, working memory, cognitive flexibility, inhibition, self-regulation, reflection, game, computer applications, videogames, preschool age 


\section{Highlights}

> The idea of training executive functions instead of forming mental tools of control over behavior underlies the majority of the existing and applied educational programs. This determines the lack of transferring skills to other activities.

- The most promising direction is creating the videogame methods which are able to adapt to a preschooler's development level and create the zone of proximal development.

- Many applications and educational programs involve parents or teachers in the development of executive functions. However, the approach involving children's cooperation with each other, when a child is a teacher for another one ("LearningBy-Teaching»), is not less promising.

For citation: Veraksa A.N., Bukhalenkova D. A. Computer game-based technology in the development of preschoolers' executive functions. Rossiiskii psikhologicheskii zhurnal - Russian Psychological Journal, 2017, V. 14, no. 3, pp. 106-132 (in Russian).

Original manuscript received 08.06.2017

\section{Введение}

Одним из самых важных достижений старшего дошкольного возраста и предиктором успешной адаптации и обучения в школе является развитие произвольности или регуляторных функций $[1,2,3,4,5]$. В связи с этим особое место в психологии дошкольного возраста занимают диагностика и создание различных методов развития когнитивной и поведенческой регуляции детей.

Современные дети посвящают значительное количество времени видеоиграм на различных компьютерных устройствах [6], что можно использовать не только для развлечения, но и для их обучения и развития. Компьютер становится все более популярен как средство развития детей среди родителей [6], а также среди педагогов и психологов [7, 8, 9, 10]. Поэтому одним из наиболее активно развивающихся направлений в современной когнитивной психологии является изучение влияния гаджетов на развитие детей различных возрастов и попытки использовать современные технологии для формирования у них различных навыков и умений. В данной статье мы хотели бы осветить зарубежные исследования, посвященные изучению влияния компьютерных игровых технологий на развитие когнитивной регуляции у детей дошкольного возраста, а также рассмотреть основные принципы создания развивающих приложений.

\section{Теоретическое обоснование}

В современной зарубежной психологии под регуляторными функциями (далее по тексту - РФ) понимается группа когнитивных навыков, 
обеспечивающих целенаправленное решение задач и адаптивное поведение в новых ситуациях. Наиболее популярной на данный момент является модель РФ А. Мияке [11], в которой выделяются три основных компонента: рабочая память, гибкость внимания, или переключение, и сдерживающий контроль. Эти компоненты связаны друг с другом, но также они могут рассматриваться как самостоятельные, отдельно друг от друга, поэтому данная модель получила название «единство с разнообразием» («unity-with-diversity»). Несмотря на то, что данная модель была изначально основана на результатах, полученных на взрослых, возможность ее использования при описании развития в детском возрасте также была подтверждена в работах зарубежных и отечественных исследователей $[12,13,14]$. Также многие ученые относят к регуляторным функциям процессы планирования и контроля поведения [15].

Согласно нейропсихологическим исследованиям, РФ напрямую связаны с развитием мозга. Постепенное развитие РФ происходит под влиянием опыта людей: созревание префронтальной коры осуществляется в условиях взаимодействия с окружающей средой, благодаря чему формуются нейронные сети, обеспечивающие системы РФ $[16,17,18]$. По этой причине тренировка конкретных познавательных способностей в детстве и подростковом возрасте способствует развитию и улучшению мозговой нейротрансмиссии, что проявляется на поведенческом уровне. Это позволяет предположить, что компьютерные игры могут стать хорошим способом тренировки, а, значит, и развития РФ в дошкольном возрасте.

Как показали классики отечественной и зарубежной психологии, наиболее значимой деятельностью в дошкольном возрасте является именно игровая деятельность [3, 19]. Ее важнейшей отличительной характеристикой является то, что в игре ребенок создает мнимую ситуацию, которая заключает в себе определенные правила поведения. Важно отметить, что игровые правила создаются самим ребенком для самого себя. Они вытекают из смысла создаваемой им воображаемой ситуации, что отличает их от правил поведения, транслируемых взрослыми. Именно в игре ребенок учится «действовать по линии наибольшего сопротивления» [19, с. 216]: подчиняться правилам, отказываться от импульсивных действий, непосредственных желаний, что доставляет ему наибольшее удовольствие. Таким образом, мы видим, что в игре развивается такой компонент РФ, как сдерживающий контроль. Как писал Л. С. Выготский, «действие в воображаемом поле, в мнимой ситуации, создание произвольного намеренья, образование жизненного плана, волевых мотивов - все это возникает в игре и ставит ее на высший уровень развития, возносит ее на гребень волны, делает ее девятым валом развития дошкольного возраста» [19, с. 220].

Таким образом, игра в дошкольном возрасте имеет ключевое значение для развития познавательных процессов, личностно-эмоциональной сферы 
и регуляции поведения. В связи с этим многие ученые стремятся использовать основные игровые принципы для создания развивающих видеоигр и приложений, направленных на улучшение когнитивных навыков [15, 20, $21,22]$. Рассмотрим подробнее, какие задачи пытаются решить исследователи с помощью создания развивающих игровых приложений.

\section{Результаты}

Причины популярности игровых методов в диагностике и коррекции когнитивных процессов

Можно выделить несколько причин, по которым именно игровые методы диагностики и коррекции являются наиболее востребованными в современной психологической практике [23].

Во-первых, использование игровых приемов позволяет повысить мотивацию и вовлеченность ребенка в процесс выполнения задания. В игре ребенок может почувствовать свою эффективность благодаря получению ярких вознаграждений за выполнение заданий - это помогает детям с трудностями когнитивной регуляции сохранять интерес и быть более внимательными. В исследовании Г. Хавкинс и коллег [24] было проведено сравнение двух версий монотонных тренировочных заданий для детей, одна из которых была представлена в обычном виде, а вторая имела черты видеоигры. В результате проведенного анализа было установлено, что видеоформат задания был более интересен испытуемым и доставлял больше удовольствия, однако, важно отметить, что это не сказалось на успешности выполнения заданий. Таким образом, исследование, с одной стороны, подтверждает идею о том, что формат видеоигры способствует большей мотивации, а с другой - показывает, что это не всегда влияет на эффективность подобных тренировочных упражнений.

Во-вторых, использование компьютерных игровых методов позволяет разрабатывать интуитивно понятные правила и адаптировать задания в соответствии с особенностями той группы, для которой они предназначены: детей [20, 25, 26], людей пожилого возраста [27], пациентов с тяжелыми нарушениями работы мозга (см. исследование В. Монтани и коллег [21]). Это крайне важно, поскольку помогает снизить тревожность, которая часто негативно сказывается на мотивации и концентрации при выполнении заданий.

B-третьих, в последние годы было проведено множество исследований, показавших положительное влияние компьютерных игр на развитие когнитивных способностей у людей разного возраста. Большинство исследований посвящены диагностике и сравнению развития познавательных процессов у подростков и юношей, увлеченных видеоиграми («геймеров»), и не играющих в них $[28,29,30,31,32]$. Например, геймеры успешнее находят необходимый 
объект в зрительных заданиях [33, 34], им легче удается подавлять лишнюю, отвлекающую информацию [35, 36], и у них в целом больше доступный объем внимания и скорость переработки информации [37]. Кроме того, существуют данные о положительном влиянии видеоигр в жанре «экшн» с элементами стратегии на развитие когнитивного контроля [38, 39, 40, 41].

Существует также небольшое количество данных о влиянии использования компьютера в дошкольном возрасте. Проведенные в последнее десятилетие отечественные исследования показали положительное влияние использования обучающих компьютерных игр для развития понятийного и практического мышления [7, 9], воображения и творческих способностей [8] у детей дошкольного возраста. В зарубежных исследованиях также было показано, что дети, имевшие доступ к компьютеру в дошкольном возрасте, отличались более высокой готовностью к школе и когнитивным развитием, при условии контроля их социоэкономического статуса $[42,43]$. Несколько исследований показали позитивное влияние использования компьютера в раннем возрасте на освоение языка $[44,45]$, а также познавательную мотивацию $[46,47]$.

Важно отметить, что полученные в подобных исследованиях данные довольно часто противоречат друг другу $[20,23]$. Такие противоречия могут быть объяснены тем, что многие исследования, сравнивающие людей, активно играющих в видеоигры и не имеющих опыта в этом, не обладают достаточной валидностью: в них часто отсутствует контроль многих факторов, нет четкого разделения между тестируемыми группами, отсутствует предварительное тестирование испытуемых перед применением игровых технологий, а только констатируется итоговое различие между ними $[28,48]$. Кроме того, подобные исследования не дают ответа на вопрос о том, какие именно черты коммерческих компьютерных игр оказывают констатируемое развивающее воздействие. Тем не менее, данные исследования еще раз указывают на возможность применения игровых методов для тренировки когнитивных навыков.

В-четвертых, разработка развивающих видеоигр помогает сделать тренировочные задания более реалистичными и, следовательно, более экологически валидными. Такой подход, по мнению ряда авторов, помогает решить проблему переноса навыков, полученных в игре, на реальную жизнь $[49,50]$. Как пишут Н. Дунбар с коллегами [51], игры отлично подходят для некоторых форм когнитивной тренировки, поскольку дают свободу игроку сделать выбор и получить обратную связь о последствиях своего выбора; иными словами, они предоставляют возможности для обучения опытным путем.

Все вышеперечисленные факторы являются несомненными достоинствами применения игровых методов. Рассмотрим, насколько широкое применение они получили в развитии и коррекции РФ у дошкольников. 


\section{Коррекция РФ у дошкольников игровыми методами}

Наиболее остро проблема саморегуляции проявляется именно в дошкольном возрасте. Многочисленные исследования показали, что дети, испытывающие сложности в саморегуляции, добиваются меньших успехов в учебе и часто испытывают отвержение со стороны сверстников в детском саду $[52,53]$. Низкий уровень саморегуляции обычно проявляется в гиперактивности, невнимательности, импульсивности, эмоциональной лабильности и часто связан с плохо развитой когнитивной регуляцией $[53,54]$. Для коррекции данных проблем за последнее десятилетие было разработано множество обучающих и развивающих программ, среди которых можно выделить несколько основных направлений.

Одно из направлений основывается на развитии гармоничного детскородительского взаимодействия, которое включает в себя обучение развивающим воспитательным стратегиям, использованию различных развивающих совместных игр. Сюда можно отнести: программу домашнего обучения для всей семьи «New Forest Parenting Package» [55]; программы по вовлечению родителей в процесс обучения их детей в школе и дома «ParentCorps» [56] и «Head Start» [57]; программу, основанную на коррекции поведенческих, эмоциональных и нейрокогнитивных функций, «Enhancing Neurobehavioral Gains with the Aid of Games and Exercise» (ENGAGE) [58]. Данные развивающие программы доказали свою эффективность, однако сами авторы признают, что при использовании такого подхода трудно сказать, что же оказывает большее влияние на развитие ребенка: взаимодействие с родителем или же тренировка когнитивных и поведенческих навыков в играх и специально разработанных упражнениях.

Другая часть развивающих программ направлена на развитие внутренних навыков саморегуляции у детей старшего дошкольного и младшего школьного возрастов. К ним можно отнести программу «Promoting Alternative Thinking Strategies» [59], которая направлена на улучшение сдерживающего контроля и речи для себя, оказывающих влияние на социоэмоциональное развитие и помогающих детям лучше контролировать свое поведение и эмоции. Также сюда можно отнести программу по развитию эмоциональной регуляции у детей старшего дошкольного и младшего школьного возрастов «Mindful Awareness Program» (MAPs) [60]. Данная программа состоит из обучающих упражнений, направленных на концентрацию на настоящем, привлечении внимания к ощущениям и опыту, получаемым здесь и сейчас. Таким образом, данные программы опять же основываются на тренировке и развитии когнитивных навыков.

Важно отметить, что применение компьютерных технологий сейчас перешло на новый уровень. Большинство детей школьного возраста обладает 
своими персональными электронными устройствами (гаджетами), что позволяет использование различных приложений, которые не обязательно должны быть связаны с играми. Так, коллектив ученых под руководством С. Шук [22] разработал приложение iSelfControl для IPad, которое позволяет ученикам с синдромом гиперактивности отслеживать и оценивать свое поведение в классе. Каждые 30 минут ребенок должен оценивать степень следования правилам, свою производительность и умение построить позитивные взаимоотношения. Учитель также дает свои оценки каждому ученику - это позволяет увидеть в итоге эффективность применения данной методики в работе с детьми. В пилотажном исследовании приняли участие 12 учеников 5 класса, посещающих специальную школу для детей с трудностями в саморегуляции, которые на протяжении 13 дней оценивали свое поведение. В результате проведенного исследования дети стали оценивать себя более объективно, и их оценки приблизились к учительским. Однако в некоторых диадах сохранялись значимые различия, которые объяснялись индивидуальными особенностями детей, что в дальнейшем будет учтено при совершенствовании приложения.

Таким образом, в программах, направленных на выработку внутренних средств саморегуляции, обычно задействованы педагоги, которые обучают детей и дают им необходимую обратную связь. В большинстве программ именно на взаимодействии с взрослым строится обучение ребенка и развитие его регуляторных навыков.

Однако существует еще один интересный подход, основанный на обучении через выполнение ребенком роли учителя («Learning-by-Teaching», или «LBT paradigm»). Взаимодействие сверстников организуется таким образом, что они становятся учителями друг для друга - это оказывает значимое влияние на развитие их когнитивных навыков [61] и самоэффективности [62]. Как показала в своих исследованиях Г. А. Цукерман, данный подход показал свою эффективность в повышении успешности усвоения учебных действий и развитии рефлексии у детей младшего школьного возраста $[63,64,65]$. Однако такой способ развития детей только начинает активно внедряться в развивающие компьютерные приложения $[66,67]$ и еще мало применялся в работе с дошкольниками [68].

Исследователи А. Аксельсон, Р. Андерсон и А. Гульз [20] представили возможности применения данного направления в работе с дошкольниками 4-6 лет на примере использования специально разработанной обучающей детской игры «Bird Hero» [25]. Проведенное ими исследование показало, что дети, показавшие достаточно низкий уровень развития РФ (а именно процессов торможения и переключения) по результатам предварительного тестирования, демонстрировали высокий уровень регуляции в процессе игры 
со сверстниками. Данное исследование показало, во-первых, возможности применения данной обучающей стратегии уже в дошкольном возрасте, а во-вторых, преимущество игровых методов перед обычными стандартизированными тестами в исследовании когнитивных возможностей детей.

Особое место в развитии РФ в дошкольном возрасте занимают специально разработанные компьютерные программы, направленные на тренировку когнитивных процессов, способствующих их развитию $[69,70,71]$. Одной из таких является программа «Cogmed» [72], направленная на развитие рабочей памяти у дошкольников. Данная программа представляет собой набор упражнений, которые дети должны выполнять на компьютере в течение 15 минут каждый день на протяжении 5 недель. Важно отметить, что, несмотря на полученные доказательства улучшения работы памяти у детей, эти достижения не влияли на эффективность выполнения других практических заданий и не отразились на поведении детей. Некоторые исследователи связывают отсутствие переноса навыка на другие стороны жизни с тем, что большинство подобных обучающих занятий и программ состоят из малоподвижных упражнений, предполагающих, что ребенок сидит на одном месте, тогда как в последнее время все больше исследований свидетельствуют о высокой значимости двигательных упражнений в развитии РФ и показывают большую эффективность активных методов по сравнению с пассивными [73, 74, $75,76]$. Так, в исследовании Дж. Беста [74], проведенном на 33 детях в возрасте 6-10 лет, было показано, что активные видеоигры (включающие в себя двигательную активность) оказывают значимое влияние на успешность выполнения заданий на РФ, тогда как обычные развивающие упражнения и пассивные видеоигры не оказали такого влияния.

Таким образом, компьютерные методы постепенно становятся все более популярными в развитии РФ, однако пока существует еще сравнительно небольшое число подобных специально разработанных игр, которые были бы направлены именно на дошкольный возраст. Тем не менее, существует несколько важных исследований, раскрывших основные принципы создания подобных игровых методик.

\section{Создание развивающих видеоигр}

В когнитивной психологии с развитием РФ чаще всего связывают игры в жанре «экшн», которые требуют от игрока хорошо развитого произвольного внимания и восприятия [40]. Интересно отметить, что экшнвидеоигры, а особенно игры-шутеры от первого лица, заставляют игрока разрабатывать различные стратегии контроля за своими действиями, чтобы оперативно реагировать на быстро движущиеся визуальные и слуховые раздражители, а также гибко адаптировать свое поведение к изменяющимся 
условиям [36, 40, 77]. Однако коммерческие видеоигры не всегда подходят в качестве инструмента нейропсихологической коррекции. Зачастую они обладают слишком сложным интерфейсом, требуют развитых когнитивных умений и скорости. Поэтому одним из самых активно развивающихся направлений в современной когнитивной психологии является создание развивающих видеоигр для детей и больных с нарушениями работы мозга.

Так, например, В. Монтани с коллегами [21] провели исследование по разработке эффективной развивающей игры, направленной на коррекцию различных нарушений работы РФ. Они выделили несколько принципов, соответствующих экологическому подходу, на которых должна базироваться развивающая видеоигра.

Во-первых, для развития когнитивной гибкости необходимо варьировать задания, чтобы стимулировать переключение испытуемого между различными когнитивными заданиями. Во-вторых, для развития объема внимания в игре необходима многозадачность. В-третьих, для развития способности к планированию и гибкому переключению между различными поведенческими стратегиями важно использовать систему усложняющихся уровней в игре. Одним из ключевых моментов в построении коррекционной игры является поддержание оптимума мотивации, который достигается путем нахождения баланса между возможностями пациента и сложностью задания.

Авторами данного исследования был разработан алгоритм, адаптирующийся под уровень пациента. Данный эффект достигался через изменение трех основных параметров игры: 1) ограничение времени на выполнение задания; 2) трудность задачи (был разработаны 9 уровней сложности); 3) особенности переключения уровней (предсказуемые или непредсказуемые). Разработанная на основе описанных принципов и алгоритма игра «Лабиринт» показала свою эффективность в работе с юношами в возрасте 19-25 лет, имеющими мозговые травмы, повлекшие за собой нарушение работы РФ. Несомненная ценность данного исследования заключается в выделении значимых особенностей видеоигр, способствующих развитию всех компонентов РФ.

В работе Т. Мандежар и коллег [15] был проведен анализ различных типов игровых действий в видеоиграх жанра «экшн», для каждого из которых были разработаны собственные игры, и выявлена их связь с работой РФ. Данная группа исследователей использовала метод ЭЭГ для измерения мозговой активности, что позволило проследить связь физиологических процессов в мозге в процессе выполнения школьниками психологической диагностики и использования видеоигр. Одним из важных достижений их работы стала классификация видеоигр, в которой определенные виды игровых действий связывались с развитием определенных регуляторных способностей детей: 
- «точные действия»: игрок должен очень аккуратно выполнить набор действий для продолжения игры (например, аккуратно водить мышкой, не задевая препятствия). Данный вид игровых действий оказался минимально связан именно с РФ, однако данный вид игр активирует практически весь мозг;

- «своевременные действия»: в этом случае игрок выполняет действие в определенный момент, который определяется событиями в игре, где скорость реакции - это самое главное (например, когда персонаж бежит по дороге и должен вовремя перепрыгивать препятствия). Данный вид игровых действий оказался в наибольшей степени связан с когнитивной гибкостью и рабочей памятью;

- «повторение последовательности действий»: игроку нужно воспроизвести последовательность действий, ранее показанную в игре (явно или скрыто) (например, ребенку показывают последовательность из нескольких картинок, которую он потом должен в точности повторить). Данный вид игровых действий показал отсутствие явной связи с РФ, однако подобные могут быть использованы для развития рабочей памяти и процессов торможения;

- «запоминание образцов действий»: игрок должен выучить схему действий, необходимую для прохождения задания при определенных событиях в игре (например, персонаж игры встречает разные препятствия на своем пути (яму, речку, врага), для преодоления которых нужно воспроизвести особую комбинацию действий). Данный вид игровых действий оказался в большей степени связан с выполнением теста Струпа, отражающего уровень развития процессов когнитивного торможения;

- «логические задачи»: в этом случае игрок должен понимать логику действий персонажа, чтобы идти вперед (например, прохождение персонажем многоуровневого лабиринта). Данный вид игровых действий значимо коррелировал с выполнением задания «Ханойская башня», которое отражает развитие процессов планирования, контроля и решения проблем. Подобные игры способствуют активации различных областей мозга и способствуют развитию всех компонентов РФ.

Таким образом, согласно данному исследованию, наибольшей пользой для развития и тренировки различных компонентов РФ обладают игры, в которых ребенок должен быстро реагировать, запоминать и повторять определенные образцы действий, а также решать логические задачи.

Полученные в данной работе результаты хорошо согласуются с результатами предыдущего исследования: в обоих подчеркивается значение заучивания испытуемым определенных наборов действий и применение их в соответствующей ситуации, заданий на скорость. Выделенные в исследованиях 
принципы могут быть использованы как в оценке имеющихся приложений и игр, так и в разработке новых развивающих игр для детей. Важно подчеркнуть, что авторы обеих работ исходят из идеи, что именно тренировка когнитивных навыков способствует развитию РФ, однако такой подход не позволяет ответить на вопрос о том, будет ли перенос полученных навыков на другие учебные действия и реальную жизнь.

Кроме того, при создании развивающих видеоигр важно находить правильный баланс между всеми компонентами игры и их влиянием на когнитивные процессы играющего [78, 79]. Исследование, проведенное Б. Катц и коллегами [78], показало, что наибольший развивающий эффект достигается такими вариациями видеоигр, в которых использовано минимальное количество игровых спецэффектов. Этого оказывается достаточно для повышения мотивации детей, тогда как добавление лишних визуальных стимулов может их только отвлекать или же повышать уровень стресса (например, когда на экране постоянно идет подсчет полученных в игре очков). В исследовании А. Миранды и Е. Палмера [79] были использованы два варианта вознаграждения ребенка за быстрые и точные ответы: звуковой сигнал и дополнительные очки. Они обнаружили, что звуковые сигналы уменьшают скорость реакции, поскольку отвлекают играющего и не повышают его вовлеченность в процесс выполнения задания; тогда как начисление бонусных баллов не влияет на эффективность, однако повышает вовлеченность. Данные исследования показывают, что сфера разработки видеоигр, развивающих когнитивные способности, крайне сложна и требует тщательного анализа всех составляющих игры.

\section{Обсуждение результатов}

Таким образом, мы видим возрастающее внимание исследователей к изучению влияния видеоигр на развитие детей и к созданию обучающих компьютерных программ, направленных на развитие различных компонентов РФ [80].

Как показал проведенный нами анализ, большинство существующих и применяющихся развивающих программ основывается на идее тренировки РФ, и в дошкольном возрасте компьютерные технологии используются в основном не как самостоятельное средство развития РФ, а скорее как способ/форма сотрудничества между взрослым и ребенком. Большинство программ предполагает, что именно взрослый (родитель или воспитатель) обучает ребенка средствам регуляции своей деятельности, тогда как развивающие видеоигры служат в основном для тренировки его регуляторных навыков. Несмотря на убедительные доказательства положительного влияния подобных игр на развитие РФ со стороны нейронауки, такой механизм развития когнитивной 
регуляции с позиции культурно-исторической психологии выглядит недостаточно убедительным, поскольку представляет собой обычное научение в результате тренировки, тогда как говорить о формировании внутренних средств управления собственным поведением довольно трудно. Исключением служит подход развития через обучение, предполагающий сотрудничество детей друг с другом, в котором компьютерная игра побуждает не только активное использование всех РФ, но и развитие рефлексии у ребенка. Данный недостаток развивающих игр, на наш взгляд, может быть причиной того, что в большинстве исследований отсутствует перенос полученных развивающих эффектов на поведение или другие виды деятельности.

Тем не менее, стремительное развитие компьютерных технологий, позволяющее создание развивающих видеоигр, открывает новые возможности перед исследователями РФ по созданию гибких развивающих средств, способных адаптироваться под уровень развития и психологические особенности испытуемого. С помощью использования разных игровых условий (временных ограничений, разных уровней сложности и разнообразия заданий) и видов действий в игре (на скорость, на точность, на запоминание последовательности действий или же логику) возможно создание такого приложения, которое будет развивать все или только определенные компоненты РФ.

В заключение хотелось бы подчеркнуть, что для большего развивающего эффекта подобных приложений важно использовать более открытые задания, которые не полностью контролируют действия играющего ребенка, а дают ему больше свободы выбора и самовыражения, что будет способствовать развитию у него рефлексии и способности к прогнозированию своей деятельности.

\section{Литература}

1. Выготский Л. С. Собрание сочинений: в 6 т. Т. 4. Детская психология / под. ред. Д. Б. Эльконина. - М. : Педагогика, 1984. - 432 с.

2. Смирнова Е.О. К проблеме воли и произвольности в культурно-исторической психологии // Культурно-историческая психология. - 2015. - Т. 11, № 3. - C. 9-15. - DOI: 10.17759/chp.2015110302

3. Willoughby M. T., Kupersmidt J. B, Voegler-Lee M. E. Is preschool executive function causally related to academic achievement? // Child Neuropsychology: A Journal on Normal and Abnormal Development in Childhood and Adolescence. - 2012. - Vol. 18 (1). - P. 79-91.

4. Yeniad N., Malda M., Mesman J., van IJzendoorn M. H., Pieper S. Shifting ability predicts math and reading performance in children: A meta-analytical study // Learning and Individual Differences. - 2013. - Vol. 23. - P. 1-9. DOI: 10.1016/j.lindif.2012.10.004 
5. Собкин В. С., Скобельцына К. Н. Представления родителей об особенностях общения их ребенка с компьютером // Современное дошкольное образование. Теория и практика. - 2012. - № 3. - С. 30-34.

6. Батенова Ю.В. Особенности развития мышления дошкольника в условиях игровой компьютерной деятельности // Психология. Журнал Высшей школы экономики. - 2011. - Т. 8, № 4. - С. 59-72.

7. Габдуллина 3. М. Развитие навыков работы с компьютером у детей 4-7 лет. - В. : Учитель, 2011. - 140 с.

8. Новоселова С. Л., Петку Г.П. Компьютерный мир дошкольника. - М. : Новая школа, 1997. - 128 с.

9. Смирнова Е. О., Радева Р.Е. Психологические особенности компьютерных игр: новый контекст детской субкультуры // Образование и информационная культура. Социологические аспекты. Труды по социологии образования. Том V. Выпуск VII. / под ред. В. С. Собкина. - М. : Центр социологии образования РАО, 2000. - С. 330-369.

10. Miyake A., Friedman N.P., Emerson M. J., Witzki A. H., Howerter A., Wager T. The unity and diversity of executive functions and their contributions to complex «frontal lobe» tasks: A latent variable analysis // Cognitive Psychology. - 2000. - Vol. 41. - P. 49-100.

11. Алмазова О. В., Бухаленкова Д. А., Веракса А. Н. Произвольность в дошкольном возрасте: сравнительный анализ различных подходов и диагностического инструментария // Национальный психологический журнал. - 2016. - 4 (24). - С. 14-22.

12. Diamond A., Lee K. Interventions shown to aid executive function development in children 4-12 years old // Science. - 2011. - Vol. 333. - P. 959-964.

13. Lehto J., Juujarvi P., Kooistra L., Pulkkinen L. Dimensions of executive functioning: evidence from children // The British Journal of Developmental Psychology. - 2003. - Vol. 21. - P. 59-80.

14. Mondéjar T., Hervás R., Johnson E., Gutierrez C., Latorre J. M. Correlation between Videogame Mechanics and Executive Functions through EEG analysis // Journal of Biomedical Informatics. - 2016. - Vol. 63. - P. 131-140.

15. Лурия А. Р. Основы нейропсихологии. - М. : Академия, 2002. - 381 с.

16. Эльконин Д. Б. Психология игры. - М. : Педагогика, 1976. - 304 с.

17. Luciana M., Nelson C. A. The functional emergence of prefrontally-guided working memory systems in four to eight year-old children // Neuropsychologia. - 1998. - Vol. 36 (3). - P. 273-293.

18. Sastre-Riba S. Early development and learning conditions: the role of the executive functions // Revue Neurologique. - 2006. - Vol. 42 (2). - P. 43-51.

19. Выготский Л. С. Игра и ее роль в психологическом развитии ребенка // Психология развития ребенка. - М. : Смысл, Эксмо, 2004. - 512 с. 
20. Axelsson A., Andersson R., Gulz A. Scaffolding executive function capabilities via play-\&-learn software for preschoolers // Journal of Educational Psychology. - 2016. - Vol. 108 (7). - P. 969-981.

21. Montani V., De Filippo De Grazia M., Zorzi M. A new adaptive videogame for training attention and executive functions: design principles and initial validation // Frontiers in Psychology. - 2014. - Vol. 5 (409). - DOI: 10.3389/ fpsyg.2014.00409

22. Schuck S., Emmerson N., Ziv H., Collins P., Arastoo S., Warschauer M., et al. Designing an iPad App to Monitor and Improve Classroom Behavior for Children with ADHD: iSelfControl Feasibility and Pilot Studies // PLoS ONE. - 2016. - Vol. 11 (10), e0164229. - DOI: 10.1371/journal.pone.0164229

23. Lumsden J., Edwards E. A., Lawrence N. S., Coyle D., Munafò M. R. Gamification of Cognitive Assessment and Cognitive Training: A Systematic Review of Applications and Efficacy // JMIR Serious Games. - 2016. - Vol. 4 (2), e11. DOI: 10.2196/games.5888

24. Hawkins G. E., Rae B., Nesbitt K. V., Brown S. D. Gamelike features might not improve data // Behavioral Research Methods. - 2013. - Vol. 45 (2). - P. 301-318.

25. Anderberg E., Axelsson A., Bengtsson S., Håkansson M., Lindberg L. Exploring the use of a teachable agent in a mathematical computer game for preschoolers // Balkenius C., Gulz A., Haake M., Johansson B. (Eds.) Intelligent, socially oriented technology. - 2013. - Vol. 154. - P. 161-171.

26. Holmes J., Gathercole S. E., Dunning D. L. Adaptive training leads to sustained enhancement of poor working memory in children // Developmental Science. - 2009. - Vol. 12. - P. F9-F15. - DOI: 10.1111/j.1467-7687.2009.00848.x

27. Sirály E., Szabó Á., Szita B., Kovács V., Fodor Z., Marosi C., et al. Monitoring the early signs of cognitive decline in elderly by computer games: an MRI study // PLoS ONE. - 2015. - Vol. 10 (2), e0117918. - DOI: 10.1371/journal. pone.0117918

28. Boot W. R., Blakely D. P., Simons D. J. Do action video games improve perception and cognition? // Frontiers in Psychology. - 2011. - Vol. 2, 226. - P. 1-6. DOI: $10.3389 /$ fpsyg.2011.00226

29. Hubert-Wallander B., Green C. S., Bavelier D. Stretching the limits of visual attention: the case of action videogames // Wiley Interdisciplinary Reviews: Cognitive Science. - 2011a. - Vol. 2. - P. 222-230. - DOI: 10.1002/wcs. 116

30. Kulikova T. I., Maliy D. V. The correlation between a passion for computer games and the school performance of younger schoolchildren // Psychology in Russia: State of the Art. - 2015. - Vol. 8 (3). - P. 124-136.

31. Latham A. J., Patston L. L., Tippett L.J. The virtual brain: 30 years of video-game play and cognitive abilities // Frontiers in Psychology. - 2013. - Vol. 4 (629). P. 1-10. - DOI: 10.3389/fpsyg.2013.00629 
32. Spence I., Feng J. Videogames and spatial cognition // Review of General Psychology. - 2010. - Vol. 14. - P. 92-104. - DOI: 10.1037/a0019491

33. Hubert-Wallander B., Green C. S., Sugarman M., Bavelier D. Changes in search rate but not in the dynamics of exogenous attention in action videogame players // Attention, Perception, \& Psychophysics. - 2011 b. - Vol. 73. P. 2399-2412. - DOI: 10.3758/s13414-011-0194-7

34. West G. L., Stevens S. A., Pun C., Pratt J. Visuospatial experience modulates attentional capture: evidence from action videogame players // Journal of Visualization. - 2008. - Vol. 8. - pp. 1-9. - DOI: 10.1167/8.16.13

35. Mishra J., Zinni M., Bavelier D., Hillyard S. A. Neural basis of superior performance of action videogame players in an attention-demanding task // Journal of Neuroscience. - 2011. - Vol. 31. - P. 992-998. - DOI: 10.1523/ JNEUROSCI.4834-10.2011

36. Wu S., Cheng C. K., Feng J., D'Angelo L., Alain C., Spence I. Playing a first-person shooter videogame induces neuroplastic change // Journal of Cognitive Neuroscience. - 2012. - Vol.24. - P. 1286-1293. - DOI: 10.1162/jocn_a_00192

37. Dye M. W., Green C. S., Bavelier D. The development of attention skills in action videogame players // Neuropsychologia. - 2009a. - Vol. 47. - P. 1780-1789. DOI: 10.1016/j.neuropsychologia.2009.02.002

38. Cain M. S., Landau A. N., Shimamura A. P. Action videogame experience reduces the cost of switching tasks // Attention, Perception, \& Psychophysics. - 2012. - Vol. 74. - P. 641-647. - DOI: 10.3758/s13414-012-0284-1

39. Chiappe D., Conger M., Liao J., Caldwell J.L., Vu K. P. L. Improving multi-tasking ability through action video games // Applied Ergonomics. - 2013. - Vol. 44. P. 278-284. - DOI: 10.1016/j.apergo.2012.08.002

40. Colzato L. S., van Leeuwen P.J. A., van den Wildenberg W. P.M., Hommel B. DOOM'd to switch: superior cognitive flexibility in players of first shooter games // Frontiers in Psychology. - 2010. - Vol. 1, 8. - P. 1-5. - DOl: 10.3389/fpsyg.2010.00008

41. Strobach T., Frensch P. A., Schubert T. Videogame practice optimizes executive control skills in dual-task and task switching situations // Acta Psychologica. 2012. - Vol. 140, Issue 1. - P. 13-24. - DOI: 10.1016/j.actpsy.2012.02.001

42. Fish A. M., Li X., McCarrick K., Butler S. T., Stanton B., Brumitt G. A., Bhavnagri N. P., Holtrop T., Partridge T. Early Childhood Computer Experience and Cognitive Development among Urban Low-Income Preschoolers // Journal of Educational Computing Research. - 2008. - Vol. 38 (1). - P. 97-113.

43. Li X., Atkins M. S. Early childhood computer experience and cognitive and motor development // Pediatrics. - 2004. - Vol. 113. - P. 1715-1722.

44. Chera P., Wood C. Animated multimedia'talking books' can promote phonological awareness in children beginning to read // Learning and Instruction. - 2003. - Vol. 13. - P. 33-52. 
45. Din F. S., Calao J. The effects of playing educational video games on kindergarten achievement // Child Study Journal. - 2001. - Vol. 31. - P. 95-102.

46. Bergin D. A., Ford M. E., Hess R. D. Patterns of motivation and social behavior associated with microcomputer use of young children // Journal of Educational Psychology. - 1993. - Vol. 85 (3). - P. 437-445.

47. Liu M. An Exploratory Study of How Pre-Kindergarten Children Use the Interactive Multimedia Technology: Implications for Multimedia Software Design // Journal of Computing in Childhood Education. - 1996. - Vol. 7 (1). - P. 71-92.

48. Green C. S., Bavelier D. Learning, attentional control, and action video games // Current Biology. - 2012. - Vol. 22. - P. R197-R206.

49. Burgess P., Alderman N., Forbes C., Costello A., Coates L., Dawson D., et al. The case for the development and use of "ecologically valid" measures of executive function in experimental and clinical neuropsychology // Journal of the International Neuropsychological Society. - 2006. - Vol. 12 (2). - P. 4-209. DOI: $10.1017 /$ S1355617706060310

50. Melby-Lervåg M., Hulme C. Is working memory training effective? A metaanalytic review // Developmental Psychology. - 2013. - Vol. 49 (2). - P. 270-291.

51. Dunbar N., Miller C., Adame B., Elizondo J., Wilson S., Lane B., et al. Implicit and explicit training in the mitigation of cognitive bias through the use of a serious game // Computers in Human Behavior. - 2014. - Vol. 37. - P. 307-318.

52. Blair C. School readiness: Integrating cognition and emotion in a neurobiological conceptualization of children's functioning at school entry // American Psychologist. - 2002. - Vol.57. - P.111-127. - DOI: 10.1037/0003-066X.57.2.111

53. McClelland M. M., Cameron C. E. Self-regulation in early childhood: Improving conceptual clarity and developing ecological measures // Child Development Perspectives. - 2012. - Vol. 6 (2). - P. 136-142. - DOI: 10.1111/j.17508606.2011.00191.x

54. Moffitt T.E., Arseneault L., Belsky D., Dickson N., Hancox R. J., Harrington H., Caspi A. A gradient of childhood self-control predicts health, wealth, and public safety // Proceedings of the National Academy of Sciences. - 2011. Vol. 108. - P. 2693-2698. - DOI: 10.1073/pnas.1010076108

55. Abikoff H. B., Thompson M., Laver-Bradbury C., Long N., Forehand R. L., Brotman L. M., ... Sonuga-Barke E. Parent training for preschool ADHD: A randomized controlled trial of specialized and generic programs // Journal of Child Psychology and Psychiatry, and Allied Disciplines. - 2015. - Vol. 56 (6). P. 618-631. - DOI: 10.1111/jcpp.12346

56. Brotman L. M., Dawson-McClure S., Calzada E. J., Huang K.-Y., Kamboukos D., Palamar J. J., Petkova E. Cluster (School) RCT of ParentCorps: Impact on Kindergarten Academic Achievement // Pediatrics. - 2013. - Vol. 131. P. e1521-e1529. - DOI: 10.1542/peds.2012-2632 
57. Neville H. J., Stevens C., Pakulak E., Bell T. A., Fanning J., Klein S., Isbell E. Familybased training program improves brain function, cognition, and behavior in lower socioeconomic status preschoolers // Proceedings of the National Academy of Sciences. - 2013. - Vol. 110. - P. 12138-12143. - DOI: 10.1073/ pnas. 1304437110

58. Healey D. M., Halperin J. M. Enhancing Neurobehavioral Gains with the Aid of Games and Exercise (ENGAGE): Initial open trial of a novel early intervention fostering the development of preschoolers' self-regulation // Child Neuropsychology. - 2015. - Vol. 21 (4). - P. 465-480. DOI: 10.1080/09297049.2014.906567

59. Riggs N.R., Greenberg M. T., Kusché C. A., Pentz M. A. The mediational role of neurocognition in the behavioral outcomes of a social-emotional prevention program in elementary school students: Effects of the PATHS curriculum // Prevention Science. - 2006. - Vol. 7. - P. 91-102. DOI: $10.1007 / \mathrm{s} 11121-005-0022-1$

60. Flook L., Smalley S. L., Kitil M. J., Galla B. M., Kaiser-Greenland S., Locke J., Kasari C. Effects of mindful awareness practices on executive functions in elementary school children // Journal of Applied School Psychology. - 2010. - Vol. 26. P. 70-95. - DOI: 10.1080/15377900903379125

61. Bargh J. A., Schul Y. On the cognitive benefits of teaching // Journal of Educational Psychology. - 1980. - № 72. - P. 583-604.

62. Moores T. T., Chang J.C.-J., Smith D. K. Clarifying the role of self-efficacy and metacognition as predictors of performance: Construct development and test // The DATA BASE for Advances in Information Systems. - 2006. Vol. 37. - P. 125-132.

63. Романеева М. П., Цукерман Г. А., Фокина Н. Э. Роль кооперации со сверстниками в психическом развитии младших школьников // Вопросы психологии. - 1980. - № 6. - С. 109-114.

64. Цукерман Г.А. Условия развития рефлексии у шестилеток // Вопросы психологии. - 1989. - № 2. - С. 39-46.

65. Цукерман Г. А. От умения сотрудничать к умению учить себя // Психологическая наука и образование. - 1996. - № 2. - С. 27-42.

66. Pareto L., Haake M., Lindström P., Sjödén B., Gulz A. A teachable-agent-based game affording collaboration and competition: Evaluating math comprehension and motivation // Educational Technology Research and Development. - 2012. - Vol. 60. - P. 723-751.

67. Schwartz D. L., Chase C., Chin D., Oppezzo M., Kwong H., Okita S., ... Biswas G. Interactive metacognition: Monitoring and regulating a teachable agent // Hacker D., Dunlosky J., Graesser A. (Eds.) Handbook of metacognition in education. - NY : Routledge Press, 2009. - P. 340-358. 
68. Chin D., Dohmen I., Schwartz D. Young children can learn scientific reasoning with teachable agents // IEEE Transactions on Learning Technologies. 2013. - Vol. 6. - P. 248-257.

69. Ackerman P. L., Kanfer R., Calderwood C. Use it or lose it? Wii brain exercise practice and reading for domain knowledge // Psychology and Aging. 2010. - 25. - P. 753-766. - DOI: $10.1037 / a 0019277$

70. Shalev L., Tsal Y., Mevorach C. Computerized progressive attentional training (CPAT) program: Effective direct intervention for children with ADHD // Child Neuropsychology. - 2007. - Vol. 13. - P. 382-388. DOI: 10.1080/09297040600770787

71. Slagter H. A., Lutz A., Greischar L. L., Francis A. D., Nieuwenhuis S., Davis J.M., Davidson R.J. Mental training affects distribution of limited brain resources // PLoS Biology. - 2007. - Vol. 5. - P. 1228-1235. - DOI: 10.1371/ Journal.pbio.0050138

72. Thorell L. B., Lindqvist S., Bergman Nutley S., Bohlin G., Klingberg T.Training transfer effects of executive functions in preschool children // Developmental Science. - 2009. - Vol. 12. - P. 106-113. - DOI: 10.1111/j.1467-7687.2008.00745.x

73. Berwid O. G., Halperin J. M. Emerging Support for a Role of Exercise in attention deficit/hyperactivity disorder intervention planning // Current Psychiatry Reports. - 2012. - № 14. - P. 543-551. - DOI: 10.1007/s11920-012-0297-4

74. Best J.R. Exergaming immediately enhances children's executive function // Developmental Psychology. - 2012. - Vol. 48 (5). - P. 1501-1510. DOI: $10.1037 / \mathrm{a} 0026648$

75. Chaddock L., Pontifex M. B., Hillman C. H., Kramer A. F. A review of the relation of aerobic fitness and physical activity to brain structure and function in children // Journal of the International Neuropsychological Society. - 2011. - Vol. 17. - P. 975-985. - DOI: 10.1017/S1355617711000567

76. Davis C. L., Tomporowski P.D., McDowell J. E., Austin B. P., Miller P. H., Yanasak N. E., ... Naglieri J. A. Exercise improves executive function and achievement and alters brain activation in overweight children: A randomized, controlled trial // Health Psychology. - 2011. - Vol. 30. - P. 91-98. DOI: $10.1037 / \mathrm{a} 0021766$

77. Steenbergen L., Sellaro R., Stock A.-K., Beste C., Colzato L. S. Action Video Gaming and Cognitive Control: Playing First Person Shooter Games Is Associated with Improved Action Cascading but Not Inhibition // PLoS ONE. - 2015. Vol. 10 (12), e0144364. - DOI: 10.1371/journal.pone.0144364

78. Katz B., Jaeggi S., Buschkuehl M., Stegman A., Shah P. Differential effect of motivational features on training improvements in school-based cognitive training // Frontiers in Human Neuroscience. - 2014. - Vol. (242). - P. 1-10. DOI: $10.3389 /$ fnhum.2014.00242 
79. Miranda A., Palmer E. Intrinsic motivation and attentional capture from gamelike features in a visual search task // Behavioral Research Methods. 2014. - Vol. 46 (1). - P. 159-172.

80. Веракса А. Н., Васильева М.Д., Арчакова Т. О. Развитие произвольности регуляторных функций в дошкольном возрасте: обзор современных зарубежных исследований // Вопросы психологии. - 2016. № 6. - С. 150-166.

\section{References}

1. Vygotsky L.S. Child psychology. In: Sobranie sochinenii [Collection of works]. Moscow, Pedagogika Publ., V. 4, 1984. 432 p.

2. Smirnova E. O. K probleme voli i proizvol'nosti v kul'turno-istoricheskoi psikhologii. Kul'turno-istoricheskaya psikhologiya - Cultural-Historical Psychology Journal, 2015, V. 11, no. 3, pp. 9-15 (in Russian). DOI: 10.17759/ chp.2015110302

3. Willoughby M.T., Kupersmidt J. B., Voegler-Lee M. E. Is preschool executive function causally related to academic achievement? Child Neuropsychology: A Journal on Normal and Abnormal Development in Childhood and Adolescence, 2012, V. 18 (1), pp. 79-91.

4. Yeniad N., Malda M., Mesman J., Van IJzendoorn M. H., Pieper S. Shifting ability predicts math and reading performance in children: A meta-analytical study. Learning and Individual Differences, 2013, V. 23, pp. 1-9. DOI: 10.1016/ j.lindif.2012.10.004

5. Sobkin V. S., Skobel'tsyna K. N. Predstavleniya roditelei ob osobennostyakh obshcheniya ikh rebenka s komp'yuterom. Sovremennoe doshkol'noe obrazovanie. Teoriya i praktika - Preschool Education Today. Theory and Practice, 2012, no. 3, pp. 30-34 (in Russian).

6. Batenova Yu. V. Osobennosti razvitiya myshleniya doshkol'nika v usloviyakh igrovoi komp'yuternoi deyatel'nosti. Psikhologiya. Zhurnal Vysshei shkoly ekonomiki - Psychology. Journal of the Higher School of Economics, 2011, V. 8, no. 4, pp. 59-72 (in Russian).

7. Gabdullina Z. M. Razvitie navykov raboty s komp'yuterom u detei 4-7 let [Development of computer skills in children aged 4-7]. Volgograd, Uchitel' Publ., 2011. $140 \mathrm{p}$.

8. Novoselova S. L., Petku G.P. Komp'yuternyi mir doshkol'nika [Computer world of preschooler]. Moscow, Novaya shkola Publ., 1997. 128 p.

9. Smirnova E. O., Radeva R. E. Psikhologicheskie osobennosti komp'yuternykh igr: novyi kontekst detskoi subkul'tury. In: Obrazovanie i informatsionnaya kul'tura. Sotsiologicheskie aspekty. Trudy po sotsiologii obrazovaniya. Tom V. Vypusk VII [Education and information culture. Sociological aspects. Works 
on the sociology of education. Volume 5, number 7]. In ed. V. S. Sobkina. Moscow, Tsentr sotsiologii obrazovaniya RAO, 2000, pp. 330-369 (in Russian). 10. Miyake A., Friedman N. P., Emerson M. J., Witzki A. H., Howerter A., Wager T. The unity and diversity of executive functions and their contributions to complex"frontal lobe" tasks: A latent variable analysis. Cognitive Psychology, 2000, V. 41, pp. 49-100.

11. Almazova O.V., Bukhalenkova D. A., Veraksa A. N. Proizvol'nost' v doshkol'nom vozraste: sravnitel'nyi analiz razlichnykh podkhodov i diagnosticheskogo instrumentariya. Natsional'nyi psikhologicheskii zhurnal - National Psychological Journal, 2016, no. 4 (24), pp. 14-22 (in Russian).

12. Diamond A., Lee K. Interventions shown to aid executive function development in children 4-12 years old. Science, 2011, V. 333, pp. 959-964.

13. Lehto J., Juujarvi P., Kooistra L., Pulkkinen L. Dimensions of executive functioning: evidence from children. The British Journal of Developmental Psychology, 2003, V. 21, pp. 59-80.

14. Mondéjar T., Hervás R., Johnson E., Gutierrez C., Latorre J. M. Correlation between Videogame Mechanics and Executive Functions through EEG analysis. Journal of Biomedical Informatics, 2016, V. 63, pp. 131-140.

15. Luriya A. R. Osnovy neiropsikhologii [Neuropsychology Foundations]. Moscow, Akademiya Publ., 2002. 128 p.

16. El'konin D. B. Psikhologiya igry [Psychology of game]. Moscow, Pedagogika Publ., 1976. 304 p.

17. Luciana M., Nelson C. A. The functional emergence of prefrontally-guided working memory systems in four to eight year-old children. Neuropsychologia, 1998, V. 36 (3), pp. 273-293.

18. Sastre-Riba S. Early development and learning conditions: the role of the executive functions. Revue Neurologique, 2006, V. 42 (2), pp. 43-51.

19. Vygotsky L. S. Play and its role in the mental development of the child. In: Psikhologiya razvitiya rebenka [Psychology of child development]. Moscow, Smysl Publ., Eksmo Publ., 2004, pp. 200-235.

20. Axelsson A., Andersson R., Gulz A. Scaffolding executive function capabilities via play-\&-learn software for preschoolers. Journal of Educational Psychology, 2016, V. 108 (7), pp. 969-981.

21. Montani V., De Filippo De Grazia M., Zorzi M. A new adaptive videogame for training attention and executive functions: design principles and initial validation. Frontiers in Psychology, 2014, V.5 (409). DOI: 10.3389/fpsyg.2014.00409

22. Schuck S., Emmerson N., Ziv H., Collins P., Arastoo S., Warschauer M., et al. Designing an iPad App to Monitor and Improve Classroom Behavior for Children with ADHD: iSelfControl Feasibility and Pilot Studies. PLOS ONE, 2016, V. 11(10), e0164229. DOI: 10.1371/journal.pone.0164229 
23. Lumsden J., Edwards E. A., Lawrence N.S., Coyle D., Munafò M.R. Gamification of Cognitive Assessment and Cognitive Training: A Systematic Review of Applications and Efficacy. JMIR Serious Games, 2016, V. 4 (2), e11. DOI: 10.2196/games.5888

24. Hawkins G.E., Rae B., Nesbitt K.V., Brown S.D. Gamelike features might not improve data. Behavioral Research Methods, 2013, V. 45 (2), pp. 301-318.

25. Anderberg E., Axelsson A., Bengtsson S., Håkansson M., Lindberg L. Exploring the use of a teachable agent in a mathematical computer game for preschoolers. In: C. Balkenius, A. Gulz, M. Haake, B. Johansson. Intelligent, socially oriented technology, 2013, V. 154, pp. 161-171.

26. Holmes J., Gathercole S. E., Dunning D. L. Adaptive training leads to sustained enhancement of poor working memory in children. Developmental Science, 2009, V. 12, pp. F9-F15. DOI: 10.1111/j.1467-7687.2009.00848.x

27. Sirály E., Szabó Á., Szita B., Kovács V., Fodor Z., Marosi C., et al. Monitoring the early signs of cognitive decline in elderly by computer games: an MRI study. PLoS ONE, 2015, V. 10 (2), e0117918. DOI: 10.1371/journal.pone.0117918

28. Boot W. R., Blakely D.P., Simons D. J. Do action video games improve perception and cognition? Frontiers in Psychology, 2011, V. 2, 226, pp. 1-6. DOI: $10.3389 /$ fpsyg.2011.00226

29. Hubert-Wallander B., Green C.S., Bavelier D. Stretching the limits of visual attention: the case of action videogames. Wiley Interdisciplinary Reviews: Cognitive Science, 2011a, V. 2, pp. 222-230. DOI: 10.1002/wcs.116

30. Kulikova T.I., Maliy D.V. The correlation between a passion for computer games and the school performance of younger schoolchildren. Psychology in Russia: State of the Art, 2015, V. 8 (3), pp. 124-136.

31. Latham A. J., Patston L. L., Tippett L. J. The virtual brain: 30 years of videogame play and cognitive abilities. Frontiers in Psychology, 2013, V. 4 (629), pp. 1-10. DOI: 10.3389/fpsyg.2013.00629

32. Spence I., Feng J. Videogames and spatial cognition. Review of General Psychology, 2010, V. 14, pp. 92-104. DOI: 10.1037/a0019491

33. Hubert-Wallander B., Green C. S., Sugarman M., Bavelier D. Changes in search rate but not in the dynamics of exogenous attention in action videogame players. Attention, Perception, \& Psychophysics, 2011b, V. 73, pp. 2399-2412. DOI: 10.3758/s13414-011-0194-7

34. West G. L., Stevens S. A., Pun C., Pratt J. Visuospatial experience modulates attentional capture: evidence from action videogame players. Journal of Visualization, 2008, V. 8, pp. 1-9. DOI: 10.1167/8.16.13

35. Mishra J., Zinni M., Bavelier D., Hillyard S. A. Neural basis of superior performance of action videogame players in an attention-demanding task. Journal of Neuroscience, 2011, V. 31, pp. 992-998. DOI: 10.1523/JNEUROSCI.4834-10.2011 
36. Wu S., Cheng C. K., Feng J., D'Angelo L., Alain C., Spence I. Playing a firstperson shooter videogame induces neuroplastic change. Journal of Cognitive Neuroscience, 2012, V. 24, pp. 1286-1293. DOI: 10.1162/jocn_a_00192

37. Dye M.W., Green C.S., Bavelier D. The development of attention skills in action videogame players. Neuropsychologia, 2009a, V. 47, pp. 1780-1789. DOI: 10.1016/j.neuropsychologia.2009.02.002

38. Cain M. S., Landau A. N., Shimamura A.P. Action videogame experience reduces the cost of switching tasks. Attention, Perception, \& Psychophysics, 2012, V. 74, pp. 641-647. DOI: 10.3758/s13414-012-0284-1

39. Chiappe D., Conger M., Liao J., Caldwell J. L., Vu K.P. L. Improving multitasking ability through action video games. Applied Ergonomics, 2013, V. 44, pp. 278-284. DOI: 10.1016/j.apergo.2012.08.002

40. Colzato L. S., van Leeuwen P. J. A., van den Wildenberg W. P. M., Hommel B. DOOM'd to switch: superior cognitive flexibility in players of first shooter games. Frontiers in Psychology, 2010, V. 1, 8, pp. 1-5. DOI: 10.3389/ fpsyg.2010.00008

41. Strobach T., Frensch P.A., Schubert T. Videogame practice optimizes executive control skills in dual-task and task switching situations. Acta Psychology, 2012, V. 140, pp. 13-24. DOI: 10.1016/j.actpsy.2012.02.001

42. Fish A. M., Li X., McCarrick K., Butler S.T., Stanton B., Brumitt G. A., Bhavnagri N.P., Holtrop T., Partridge T. Early Childhood Computer Experience and Cognitive Development among Urban Low-Income Preschoolers. Journal of Educational Computing Research, 2008, V. 38 (1), pp. 97-113.

43. Li X., Atkins M.S. Early childhood computer experience and cognitive and motor development. Pediatrics, 2004, V. 113, pp. 1715-1722.

44. Chera P., Wood C. Animated multimedia 'talking books' can promote phonological awareness in children beginning to read. Learning and Instruction, 2003, V. 13, pp. 33-52.

45. Din F. S., Calao J. The effects of playing educational video games on kindergarten achievement. Child Study Journal, 2001, V. 31, pp. 95-102.

46. Bergin D. A., Ford M.E., Hess R.D. Patterns of motivation and social behavior associated with microcomputer use of young children. Journal of Educational Psychology, 1993, V. 85 (3), pp. 437-445.

47. Liu M. An Exploratory Study of How Pre-Kindergarten Children Use the Interactive Multimedia Technology: Implications for Multimedia Software Design. Journal of Computing in Childhood Education, 1996, V. 7 (1), pp. 71-92.

48. Green C. S., Bavelier D. Learning, attentional control, and action video games. Current Biology, 2012, V. 22, pp. R197-R206.

49. Burgess P., Alderman N., Forbes C., Costello A., Coates L., Dawson D., et al. The case for the development and use of "ecologically valid" measures of 
executive function in experimental and clinical neuropsychology. Journal of the International Neuropsychological Society, 2006, V. 12 (2), pp. 4-209. DOI: $10.1017 /$ S1355617706060310

50. Melby-Lervåg M., Hulme C. Is working memory training effective? A metaanalytic review. Developmental Psychology, 2013, V. 49 (2), pp. 270-291.

51. Dunbar N., Miller C., Adame B., Elizondo J., Wilson S., Lane B., et al. Implicit and explicit training in the mitigation of cognitive bias through the use of a serious game. Computers in Human Behavior, 2014, V. 37, pp. 307-318.

52. Blair C. School readiness: Integrating cognition and emotion in a neurobiological conceptualization of children's functioning at school entry. American Psychologist, 2002, V. 57, pp. 111-127. DOI: 10.1037/0003-066X.57.2.111

53. McClelland M. M., Cameron C.E. Self-regulation in early childhood: Improving conceptual clarity and developing ecological measures. Child Development Perspectives, 2012, V. 6 (2), pp. 136-142. DOI: 10.1111/j.1750-8606.2011.00191.x

54. Moffitt T.E., Arseneault L., Belsky D., Dickson N., Hancox R. J., Harrington H., Caspi A. A gradient of childhood self-control predicts health, wealth, and public safety. Proceedings of the National Academy of Sciences, 2011, V. 108, pp. 2693-2698. DOI: 10.1073/pnas.1010076108

55. Abikoff H. B., Thompson M., Laver-Bradbury C., Long N., Forehand R. L., Brotman L. M., ... Sonuga-Barke E. Parent training for preschool ADHD: A randomized controlled trial of specialized and generic programs. Journal of Child Psychology and Psychiatry, and Allied Disciplines, 2015, V. 56 (6), pp. 618-631. DOI: 10.1111/jcpp.12346

56. Brotman L. M., Dawson-McClure S., Calzada E.J., Huang K.-Y., Kamboukos D., Palamar J. J., Petkova E. Cluster (shool) RCT of ParentCorps: Impact on kindergarten academic achievement. Pediatrics, 2013, V. 131, pp. e1521-e1529. DOI: 10.1542/peds.2012-2632

57. Neville H. J., Stevens C., Pakulak E., Bell T. A., Fanning J., Klein S., Isbell E. Family-based training program improves brain function, cognition, and behavior in lower socioeconomic status preschoolers. Proceedings of the National Academy of Sciences, 2013, V. 110, pp. 12138-12143. DOI: 10.1073/ pnas. 1304437110

58. Healey D.M., Halperin J.M. Enhancing Neurobehavioral Gains with the Aid of Games and Exercise (ENGAGE): Initial open trial of a novel early intervention fostering the development of preschoolers' self-regulation. Child Neuropsychology, 2015, V. 21 (4), pp. 465-480. DOI: 10.1080/09297049.2014.906567

59. Riggs N. R., Greenberg M.T., Kusché C. A., Pentz M. A. The mediational role of neurocognition in the behavioral outcomes of a social-emotional prevention program in elementary school students: Effects of the PATHS curriculum. Prevention Science, 2006, V. 7, pp. 91-102. DOI: 10.1007/s11121-005-0022-1 
60. Flook L., Smalley S. L., Kitil M. J., Galla B. M., Kaiser-Greenland S., Locke J., Kasari C. Effects of mindful awareness practices on executive functions in elementary school children. Journal of Applied School Psychology, 2010, V. 26, pp. 70-95. DOI: 10.1080/15377900903379125

61. Bargh J. A., Schul Y. On the cognitive benefits of teaching. Journal of Educational Psychology, 1980, no. 72, pp. 583-604.

62. Moores T.T., Chang J.C.-J., Smith D.K. Clarifying the role of self-efficacy and metacognition as predictors of performance: Construct development and test. The DATA BASE for Advances in Information Systems, 2006, V. 37, pp. 125-132.

63. Romaneeva M.P., Tsukerman G. A., Fokina N. E. Rol' kooperatsii so sverstnikami v psikhicheskom razvitii mladshikh shkol'nikov. Voprosy psikhologii - Approaches to Psychology, 1980, no. 6, pp. 109-114 (in Russian).

64. Tsukerman G. A. Usloviya razvitiya refleksii u shestiletok. Voprosy psikhologii - Approaches to Psychology, 1989, no. 2, pp. 39-46 (in Russian).

65. Tsukerman G. A. Ot umeniya sotrudnichat' k umeniyu uchit' sebya. Psikhologicheskaya nauka i obrazovanie - Psychological Science and Education, 1996, no. 2, pp. 27-42 (in Russian).

66. Pareto L., Haake M., Lindström P., Sjödén B., Gulz A. A teachable-agent-based game affording collaboration and competition: Evaluating math comprehension and motivation. Educational Technology Research and Development, 2012, V. 60, pp. 723-751.

67. Schwartz D.L., Chase C., Chin D., Oppezzo M., Kwong H., Okita S., ... Biswas $\mathrm{G}$. Interactive metacognition: Monitoring and regulating a teachable agent. In D. Hacker, J. Dunlosky, A. Graesser, Handbook of metacognition in education. NY, Routledge Press, 2009, pp. 340-358.

68. Chin D., Dohmen I., Schwartz D. Young children can learn scientific reasoning with teachable agents. IEEE Transactions on Learning Technologies, 2013, V. 6, pp. 248-257.

69. Ackerman P.L., Kanfer R., Calderwood C. Use it or lose it? Wii brain exercise practice and reading for domain knowledge. Psychology and Aging, 2010, 25, pp. 753-766. DOI: 10.1037/a0019277

70. Shalev L., Tsal Y., Mevorach C. Computerized progressive attentional training (CPAT) program: Effective direct intervention for children with ADHD. Child Neuropsychology, 2007, V. 13, pp. 382-388. DOI: 10.1080/09297040600770787

71. Slagter H. A., Lutz A., Greischar L.L., Francis A.D., Nieuwenhuis S., Davis J.M., Davidson R. J. Mental training affects distribution of limited brain resources. PLoS Biology, 2007, V. 5, pp. 1228-1235. DOI: 10.1371/Journal.pbio.0050138

72. Thorell L. B., Lindqvist S., Bergman N.S., Bohlin G., Klingberg T. Training transfer effects of executive functions in preschool children. Developmental Science, 2009, V. 12, pp. 106-113. DOI: 10.1111/j.1467-7687.2008.00745.x 
73. Berwid O.G., Halperin J. M. Emerging Support for a Role of Exercise in attention deficit/hyperactivity disorder intervention planning. Current Psychiatry Reports, 2012, no. 14, pp. 543-551. DOI: 10.1007/s11920-012-0297-4

74. Best J.R. Exergaming immediately enhances children's executive function. Developmental Psychology, 2012, V. 48 (5), pp. 1501-1510. DOI: 10.1037/a0026648

75. Chaddock L., Pontifex M. B., Hillman C.H., Kramer A. F. A review of the relation of aerobic fitness and physical activity to brain structure and function in children. Journal of the International Neuropsychological Society, 2011, V. 17, pp. 975-985. DOI: 10.1017/S1355617711000567

76. Davis C.L., Tomporowski P.D., McDowell J. E., Austin B.P., Miller P.H., Yanasak N.E., ... Naglieri J.A. Exercise improves executive function and achievement and alters brain activation in overweight children: A randomized, controlled trial. Health Psychology, 2011, V. 30, pp. 91-98. DOI: 10.1037/a0021766

77. Steenbergen L., Sellaro R., Stock A.-K., Beste C., Colzato L. S. Action Video Gaming and Cognitive Control: Playing First Person Shooter Games Is Associated with Improved Action Cascading but Not Inhibition. PLOS ONE, 2015, V. 10 (12), e0144364. DOI: 10.1371/journal.pone.0144364

78. Katz B., Jaeggi S., Buschkuehl M., Stegman A., Shah P. Differential effect of motivational features on training improvements in school-based cognitive training. Frontiers in Human Neuroscience, 2014, V. 8 (242), pp. 1-10. DOI: $10.3389 /$ fnhum.2014.00242

79. Miranda A., Palmer E. Intrinsic motivation and attentional capture from gamelike features in a visual search task. Behavioral Research Methods, 2014, V. 46 (1), pp. 159-172.

80. Veraksa A. N., Vasil'eva M. D., Archakova T.O. Razvitie proizvol'nosti regulyatornykh funktsii v doshkol'nom vozraste: obzor sovremennykh zarubezhnykh issledovanii. Voprosy psikhologii-Approaches to Psychology, 2016, no. 6, pp. 150-166 (in Russian). 